\title{
The Long-Term Effectiveness of Trigger Point Dry Needling and Exercise for Individuals With Shoulder Pain: A Critically Appraised Topic
}

\author{
Kyle Matsel, Claire Davies, and Tim Uhl
}

\begin{abstract}
Clinical Scenario: Shoulder pain is a very common symptom encountered in outpatient physical therapy practice. In addition to therapeutic exercise and manual therapy interventions, trigger point dry needling (TDN) has emerged as a possible treatment option for reducing shoulder pain and improving function. Dry needling consists of inserting a thin stainless-steel filament into a myofascial trigger point with the intention of eliciting a local twitch response of the muscle. It is theorized that this twitch response results in reduced muscle tension and can aid in reduced pain and disability. To this point, multiple studies have found TDN to be effective at reducing pain and improving function in the short-term, but the long-term outcomes remain unknown. Clinical Question: Does the addition of TDN to an exercise program result in better long-term pain intensity and disability reduction in patients with shoulder pain? Summary of Findings: Improvement in long-term pain and function can be expected regardless of the addition of TDN to an evidence-based exercise program for patients with shoulder pain. Clinical Bottom Line: Either TDN or an evidence-based therapeutic exercise program elicits improved long-term pain and disability reduction in patients with shoulder pain, which suggests that clinicians can confidently use either approach with their patients. Strength of Recommendation: Strong evidence (level 2 evidence with PEDro scores $>8 / 10$ ) suggesting that TDN does not outperform therapeutic exercise regarding long-term pain reduction.
\end{abstract}

Keywords: shoulder complex, manual therapy

\section{Clinical Scenario}

Shoulder pain is the third most commonly encountered musculoskeletal condition in orthopedic physical therapy practice, behind only neck and low-back pain. ${ }^{1}$ Shoulder pain accounts for approximately $14 \%$ to $21 \%$ of all musculoskeletal visits, and the estimated costs approach 7 billion dollars annually in the United States. ${ }^{2,3}$ Shoulder pain involves a range of pathologies, such as subacromial impingement, rotator cuff tendonitis, instability, and subacromial bursitis. These conditions have traditionally been managed with corticosteroid injections and physical therapy intervention consisting of improving strength and flexibility. 4,5

In addition to therapeutic exercise and manual therapy interventions, trigger point dry needling (TDN) has emerged as a possible treatment option for reducing shoulder pain and functional status. ${ }^{6}$ TDN is an intervention performed by physical therapists that is defined as an intramuscular procedure involving the isolated treatment of myofascial trigger points for the management of pain and neuromusculoskeletal dysfunction. TDN is effective at immediately increasing the pressure pain threshold, improving range of motion, and decreasing musculoskeletal pain in orthopedic patients. ${ }^{7}$ Likewise, improved range of motion and decreased pain are thought to be related to reduced disability and improved function. ${ }^{8}$ However, long-term improvements in pain and function in patients treated with TDN for shoulder pain are unknown.

Matsel is with the Department of Physical Therapy, University of Evansville, Evansville, IN, USA. Davies and Uhl are with the Department of Rehabilitation Sciences, University of Kentucky, Lexington, KY, USA. Matsel (km218@ evansville.edu) is corresponding author.

\section{Focused Clinical Question}

Does the addition of TDN to an evidence-based therapeutic exercise program result in better long-term pain intensity and disability reduction in patients with shoulder pain?

\section{Summary of Search}

The search terms retrieved 21 citations; 5 duplicate studies and 8 studies focusing on the wrong intervention/body region were excluded. Eight relevant citations were retrieved; 2 studies were excluded because they were not a high enough level of evidence (case reports), and 3 studies were excluded for not containing any long-term following ( $>3 \mathrm{mo}$ ). Three studies met the inclusion/ exclusion criteria.

\section{Clinical Bottom Line}

There is strong evidence to suggest that both TDN and an evidencebased therapeutic exercise program yield similar outcomes regarding long-term pain and disability reduction in patients with shoulder pain. The studies appraised in this critically appraised topic (CAT) observed no significant difference in long-term pain reduction with or without the application of dry needling for shoulder pain. Clinicians should continue using evidence-based therapeutic exercise programs as the foundation for their treatment planning in patients with shoulder pain but may consider including TDN for added benefits, specifically with long-term functional improvements. 


\section{Strength of Recommendation}

Grade A evidence exists that TDN with exercise is no more effective than exercise alone at reducing pain long term.

\section{Search Strategy}

The databases of MEDLINE, CINAHL, and SPORTDiscus were systematically searched using the following key terms: shoulder pain AND dry needling AND function AND pain NOT neck.

- Patient/client group: adults with subacute or chronic shoulder pain, impingement, or tendinitis

- Intervention (or assessment): TDN

- Comparison: therapeutic exercise

- Outcome(s): pain, functional status

\section{Inclusion and Exclusion Criteria}

\section{Inclusion Criteria}

- Adults (>18 y old) with shoulder pain of nontraumatic origin

- TDN as the intervention

- Long-term follow-up (>3 mo)

- Published in English

- Included if written after the year 2010

\section{Exclusion Criteria}

- Studies that were case reports or expert opinion

- Subjects who had undergone surgical intervention for shoulder pain

- Studies written before 2010

\section{Results of Search}

An extensive search of the literature resulted in 21 total articles; however, only 3 met the eligibility requirements and were considered for review. These studies are summarized in Tables 1 and 2 . Two studies were randomized controlled trials that examined the effect of TDN with and without exercise. ${ }^{10,12}$ One study was a retrospective case series. ${ }^{11}$

\section{Best Evidence}

In total, 3 relevant studies ${ }^{10-12}$ that met the inclusion criteria were included in this appraisal. These studies utilized TDN and

Table 1 Summary of Study Designs of Articles Retrieved

\begin{tabular}{llcl}
\hline $\begin{array}{l}\text { Level of } \\
\text { evidence }\end{array}$ & $\begin{array}{l}\text { Study design/ } \\
\text { methodology of } \\
\text { articles retrieved }\end{array}$ & $\begin{array}{l}\text { Number } \\
\text { located }\end{array}$ & Author \\
\hline $1 \mathrm{~b}$ & $\begin{array}{l}\text { Randomized } \\
\text { controlled trial }\end{array}$ & 2 & $\begin{array}{l}\text { Arias-Buria et } \mathrm{al}^{9} \text { and } \\
\text { Perez-Palomares et al }\end{array}$ \\
& $\begin{array}{l}\text { Retrospective } \\
\text { case series }\end{array}$ & 1 & $\begin{array}{l}\text { Saylor-Pavkovich } \\
\text { et al }^{11}\end{array}$ \\
\hline
\end{tabular}

therapeutic exercise as treatment, evaluated long-term pain reduction and/or functional outcomes related to TDN, and were the most recent studies evaluating this population.

\section{Summary of Best Evidence}

\section{Implications for Practice, Education, and Future Research}

The main findings from this critical appraisal suggest that the addition of TDN to an evidence-based therapeutic exercise program is more effective at achieving long-term functional disability reduction than exercises alone. However, it appears that TDN to the shoulder muscles is not superior to therapeutic exercise specific to long-term shoulder pain reduction. Although TDN has grown in popularity in the clinical setting, long-term outcomes in pain and disability reduction of this tool have never been synthesized. To this point, most research has focused on the short-term or immediate effectiveness of the TDN as a treatment for shoulder pain. A recent systematic review by Hall et al $^{13}$ concluded that there is very low-level evidence supporting TDN to improve pain and function in patients with shoulder pain. However, it may be possible that dry needling is more applicable in specific populations. Kamali et $\mathrm{al}^{14}$ found that TDN to the infraspinatus muscle was effective at reducing pain and improving disability immediately following treatment in overhead athletes. However, no long-term follow-up was conducted. Similarly, Arias-Buria et $\mathrm{al}^{9}$ found that a single session of TDN to the shoulder led to a faster recovery of function in patients recovering from postoperative shoulder surgery. Again, these studies focused on the immediate pain and disability changes of the shoulder, with no sustainable long-term follow-up making it difficult to suggest that these effects are maintained indefinitely.

There is high-level evidence suggesting that pain relief and disability reductions can be achieved by utilizing TDN along with an exercise program for shoulder pain. ${ }^{10-12}$ However, the degree to which TDN contributes to long-term pain reduction and disability reduction is still somewhat ambiguous. Arias-Buria et $\mathrm{al}^{12}$ reported that the addition of TDN to an exercise program had a significantly greater disability reduction, which surpassed the minimal clinically important difference at 12 months compared with exercise alone. However, there were not between-group differences regarding long-term pain reduction. In a retrospective case series, SaylorPavkovich et $\mathrm{al}^{11}$ reported that functional status and pain reduction were significantly improved in a case series of 8 patients who received the combination of TDN and exercise 8 months following intervention. Conversely, Perez-Palomares et $\mathrm{al}^{10}$ found no difference in pain reduction or functional status at a 3-month follow-up in individuals who received TDN and exercise compared with only exercise. However, it should be noted that, in the randomized controlled trials by Arias-Buria et al $^{12}$ and Perez-Palomares et al, ${ }^{10}$ numerical pain rating and functional status increased similarly between those experimental and control groups (2). Besides postneedling soreness, the procedure appears to have limited adverse effects and may serve an effective addition to a high-quality therapeutic exercise program to patients expecting long-term pain relief and functional improvement.

This CAT is not without limitations. First, although this CAT included 2 randomized controlled trials, ${ }^{10,12}$ which directly compared an evidence-based therapeutic exercise to TDN, the third study was a retrospective case series ${ }^{11}$ that did not have a control group for comparison. It is possible that both therapeutic exercise and TDN are effective means of reducing pain and improving 

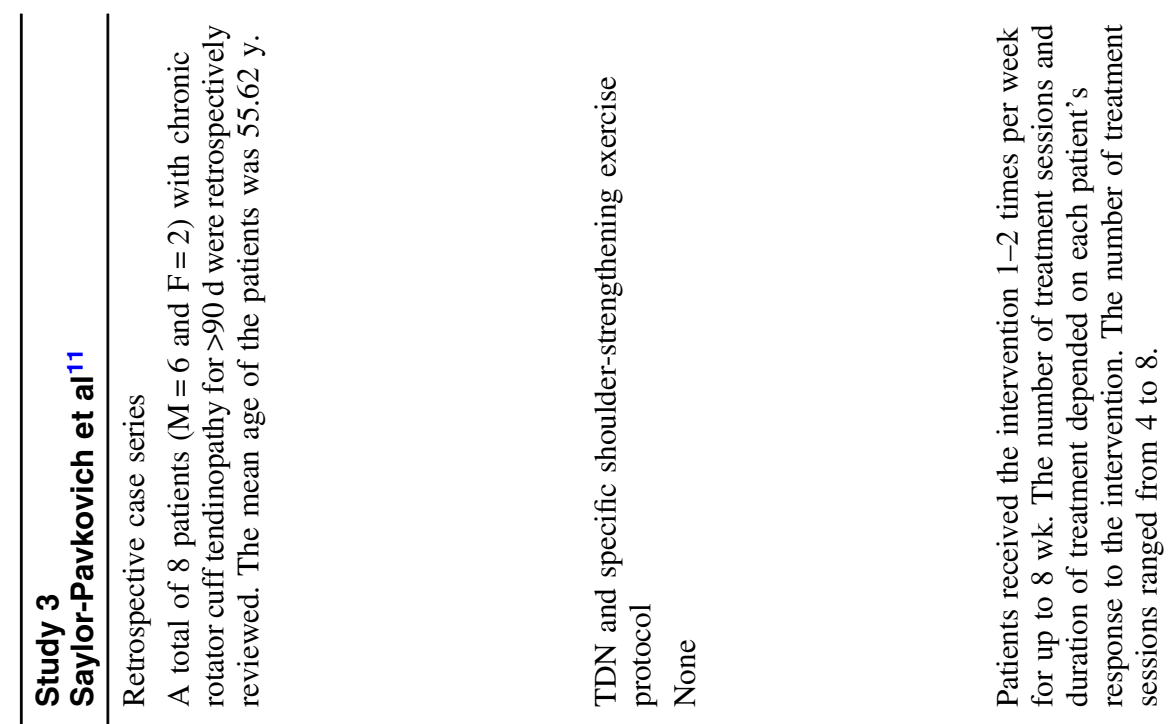

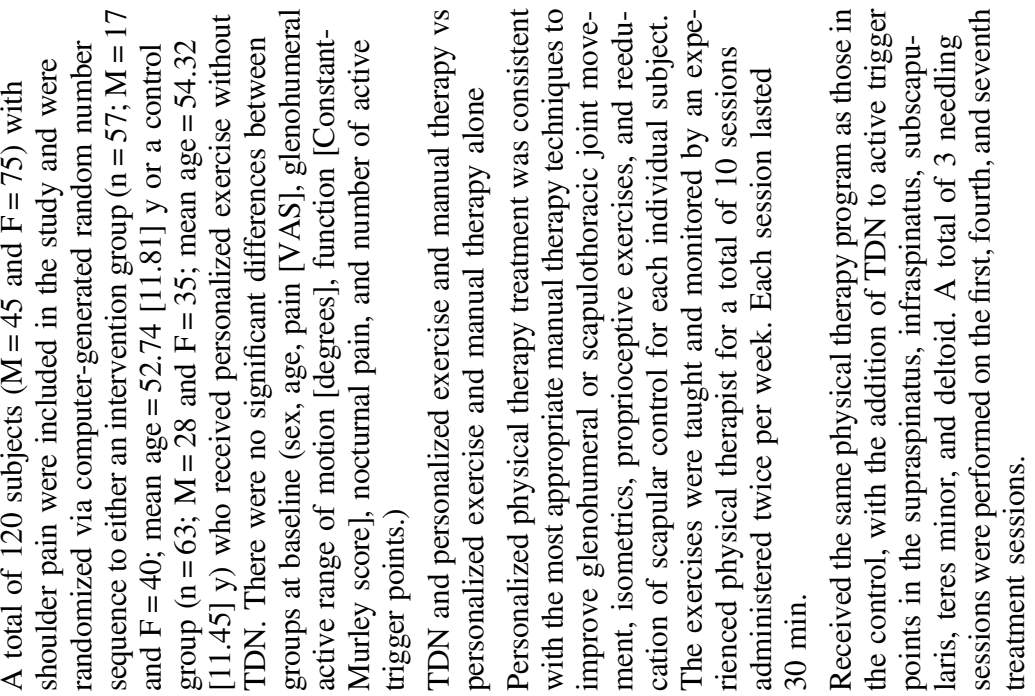

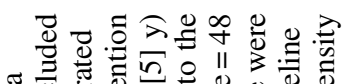

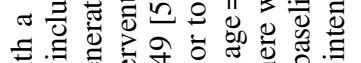

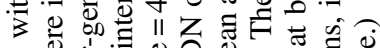

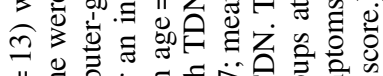

政

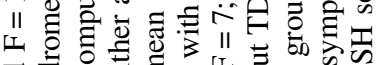

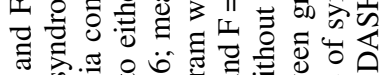

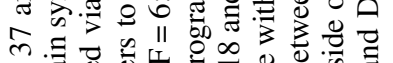

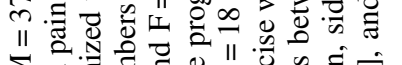

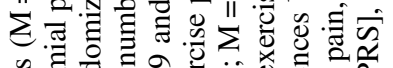

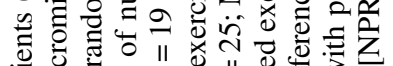

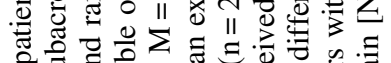

2.

蛇羊矛

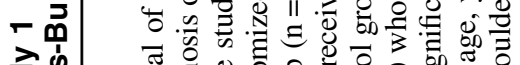

줗ㅎํ

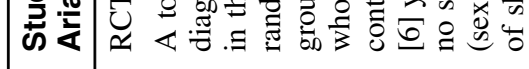

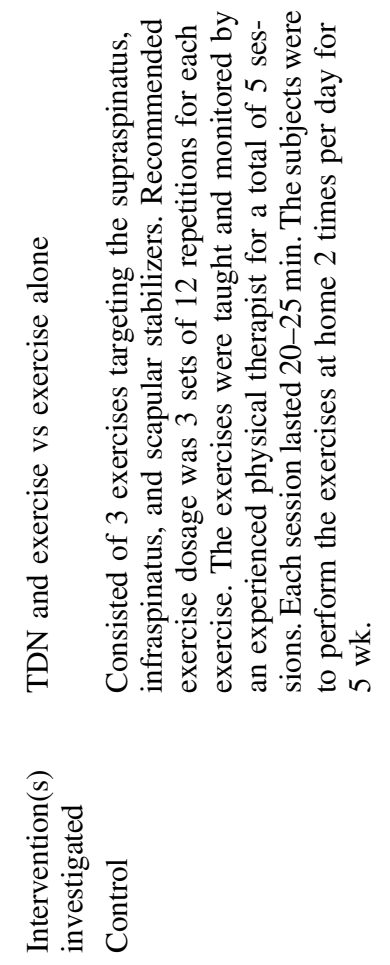

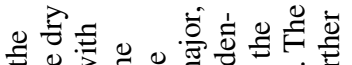

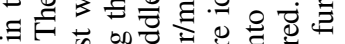

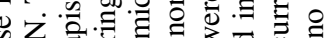

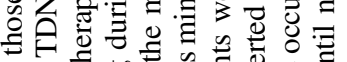

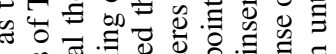

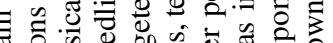
额.

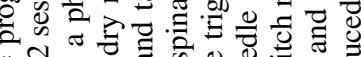
论 एँ.

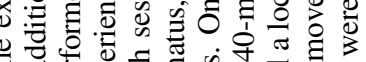

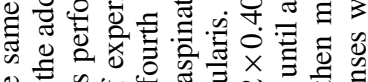

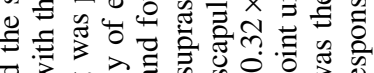
उ 3 on 2 的

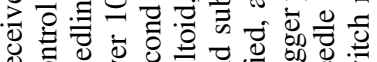
促 


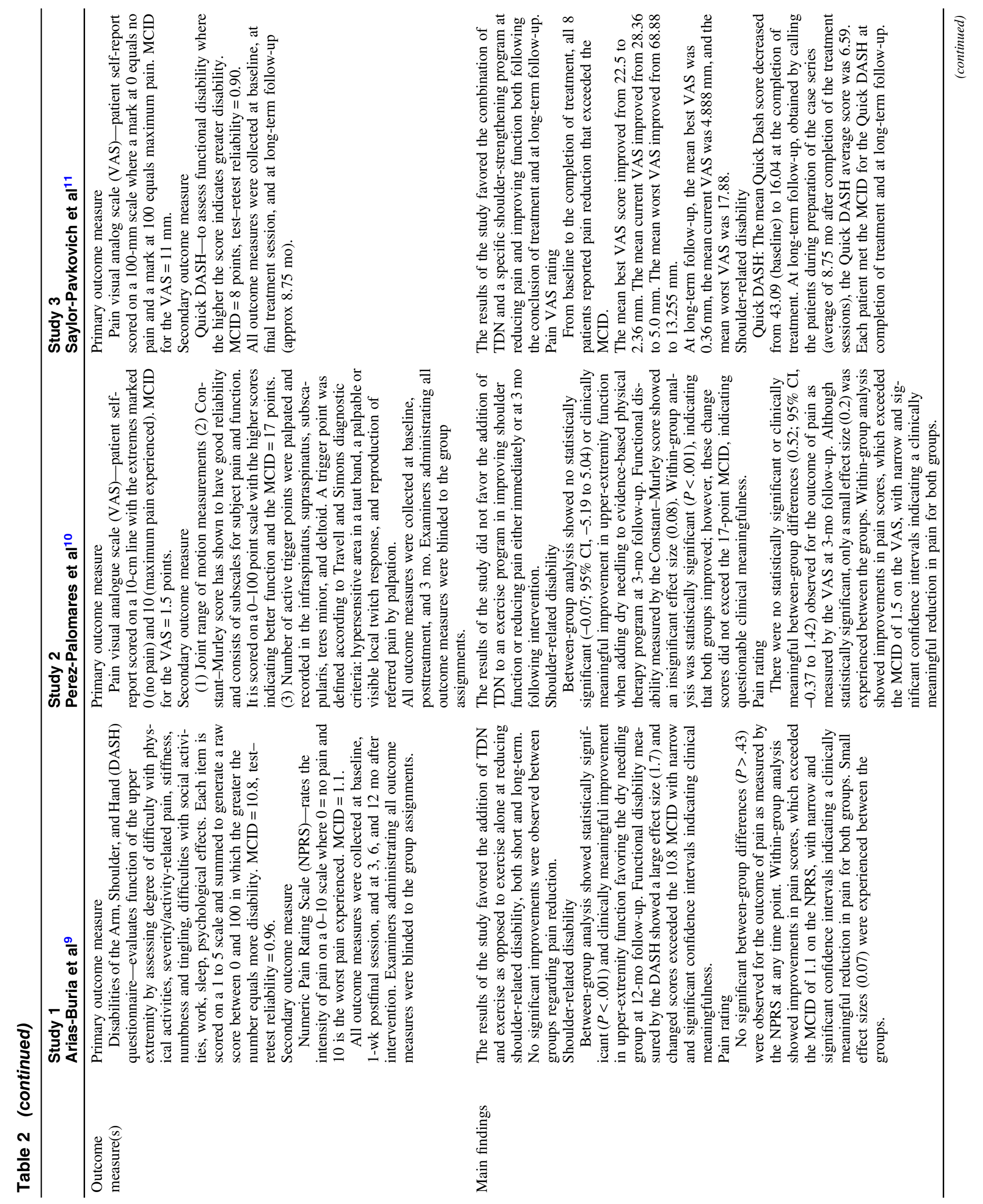




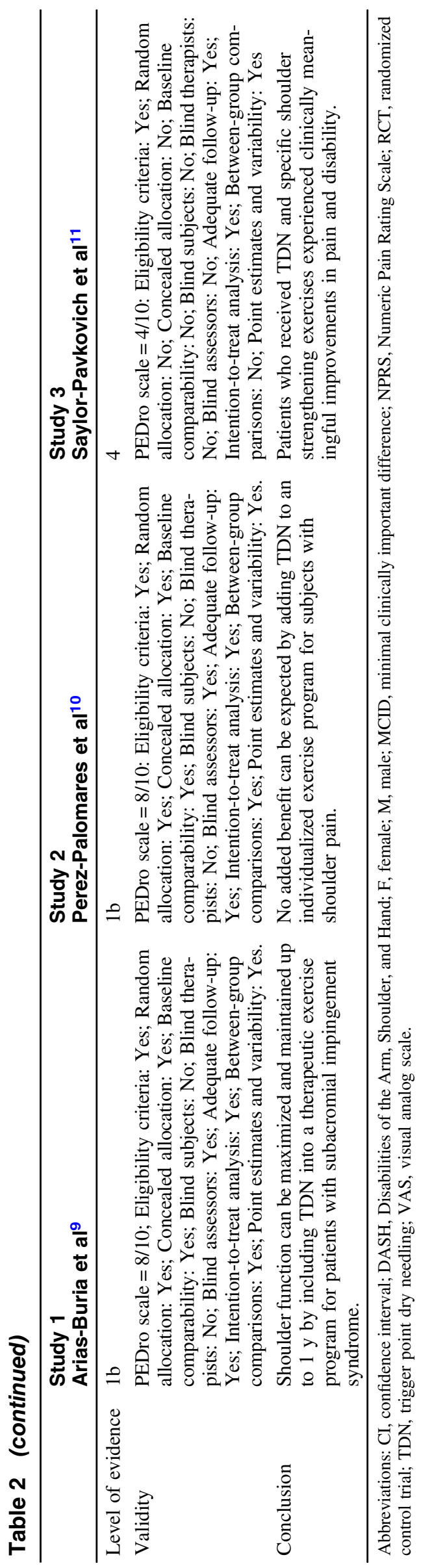

JSR Vol. 30, No. 2, 2021 
function in the shoulder, which could explain why large differences could not be detected. Second, consistency among the dosage and frequency of TDN varies. Arias-Buria et $\mathrm{al}^{12}$ only performed 2 sessions of TDN, 2 weeks apart, compared with Perez-Palmares et al, ${ }^{10}$ who recommended 3 sessions of TDN, 3 weeks apart. It is possible that the more frequent sessions of TDN over longer time would have resulted in a larger effect on the outcomes. Finally, the effects of manual therapy versus TDN need to be established. Perez-Palmares et al $^{10}$ utilized manual therapy and therapeutic exercise compared with manual therapy, exercise, and TDN. It is possible that the effects of manual therapy may have masked the improvements of TDN, resulting in no difference between groups.

Future research should include high-quality randomized trials that include a true control group to better ascertain the effectiveness of TDN. Specifically studying the effects of manual therapy and exercise compared with TDN and exercise may provide insight into which treatment modality is more effective. Furthermore, future research should explore the development of a clinical predictive rule to identify characteristics consistent with positive TDN outcomes for patients with shoulder pain. This would provide rehabilitation professionals with guidance as to when TDN is most appropriate for individuals with shoulder pain.

\section{Conclusion}

The results of this CAT suggest that the combination of TDN and exercises is just as effective as an exercise program alone at reducing long-term pain and disability. Clinicians should feel confident that TDN will not result in major adverse effects and may better improve functional outcomes compared with the implementation of exercise exclusively. At this time, more research is needed to determine which situations are best for adding TDN to a therapeutic exercise program to maximize the long-term reduction of shoulder pain and dysfunction.

\section{Acknowledgments}

The authors have no conflicts of interest to disclose.

\section{References}

1. Dinnes J, Loveman E, McIntyre L, Waugh N. The effectiveness of diagnostic tests for the assessment of shoulder pain due to soft tissue disorders: a systematic review. Health Technol Assess. 2003;7(29):iii, 1-166. doi:10.3310/hta7290

2. Luime JJ, Koes BW, Hendriksen IJ, et al. Prevalence and incidence of shoulder pain in the general population; a systematic review. Scand $J$ Rheumatol. 2004;33(2):73-81. PubMed ID: 15163107 doi:10.1080/ 03009740310004667
3. Wofford JL, Mansfield RJ, Watkins RS. Patient characteristics and clinical management of patients with shoulder pain in U.S. primary care settings: secondary data analysis of the National Ambulatory Medical Care Survey. BMC Musculoskelet Disord. 2005;6(4):4. PubMed ID: 15691370 doi:10.1186/1471-2474-6-4

4. Diercks R, Bron C, Dorrestijn O, et al. Guideline for diagnosis and treatment of subacromial pain syndrome: a multidisciplinary review by the Dutch Orthopaedic Association. Acta Orthop. 2014;85(3): 314-322. PubMed ID: 24847788 doi:10.3109/17453674.2014.920991

5. Hanratty CE, McVeigh JG, Kerr DP, et al. The effectiveness of physiotherapy exercises in subacromial impingement syndrome: a systematic review and meta-analysis. Semin Arthritis Rheum. 2012; 42(3):297-316. PubMed ID: 22607807 doi:10.1016/j.semarthrit. 2012.03.015

6. Trigger point dry needling. J Orthop Sports Phys Ther. 2017; 47(3):150. doi:10.2519/jospt.2017.0502

7. Gattie E, Cleland JA, Snodgrass S. The effectiveness of trigger point dry needling for musculoskeletal conditions by physical therapists: a systematic review and meta-analysis. J Orthop Sports Phys Ther. 2017; 47(3):133-149. PubMed ID: 28158962 doi:10.2519/jospt.2017.7096

8. Salom-Moreno J, Ayuso-Casado B, Tamaral-Costa B, Sánchez-MiláZ, Fernández-de-las-Peñas C, Alburquerque-Sendín F. Trigger point dry needling and proprioceptive exercises for the management of chronic ankle instability: a randomized clinical trial. Evid Based Complement Alternat Med. 2015;2015:790209. doi:10.1155/2015/790209

9. Arias-Buria JL, Valero-Alcaide R, Cleland JA, et al. Inclusion of trigger point dry needling in a multimodal physical therapy program for postoperative shoulder pain: a randomized clinical trial. J Manipulative Physiol Ther. 2015;38(3):179-187.

10. Perez-Palomares S, Olivan-Blazquez B, Perez-Palomares A, et al. Contribution of dry needling to individualized physical therapy treatment of shoulder pain: a randomized clinical trial. J Orthop Sports Phys Ther. 2017;47(1):11-20.

11. Saylor-Pavkovich E. Strength exercises combined with dry needling with electrical stimulation improve pain and function in patients with chronic rotator cuff tendinopathy: a retrospective case series. Int $J$ Sports Phys Ther. 2016;11(3):409-422.

12. Arias-Buria JL, Fernandez-de-Las-Penas C, Palacios-Cena M, Koppenhaver SL, Salom-Moreno J. Exercises and dry needling for subacromial pain syndrome: a randomized parallel-group trial. $J$ Pain. 2017;18(1):11-18.

13. Hall ML, Mackie AC, Ribeiro DC. Effects of dry needling trigger point therapy in the shoulder region on patients with upper extremity pain and dysfunction: a systematic review with meta-analysis. Physiotherapy. 2018;104(2):167-177. PubMed ID: 29439829 doi:10. 1016/j.physio.2017.08.001

14. Kamali F, Sinaei E, Morovati M. Comparison of upper trapezius and infraspinatus myofascial trigger point therapy by dry needling in overhead athletes with unilateral shoulder impingement syndrome. $J$ Sport Rehabil. 2018;1-24. 